Marquette University

e-Publications@Marquette

8-17-2018

\title{
Obtaining Chemical Selectivity from a Single, Nonselective Sensing Film: Two-Stage Adaptive Estimation Scheme with Multiparameter Measurement to Quantify Mixture Components and Interferents
}

\author{
Karthick Sothivelr \\ Marquette University \\ Florian Bender \\ Marquette University, florain.bender@marquette.edu \\ Fabien Josse PhD \\ Marquette University, fabien.josse@marquette.edu \\ Edwin E. Yaz \\ Marquette University, edwin.yaz@marquette.edu \\ Antonio J. Ricco \\ Stanford University
}

Follow this and additional works at: https://epublications.marquette.edu/electric_fac

Part of the Computer Engineering Commons, and the Electrical and Computer Engineering Commons

\section{Recommended Citation}

Sothivelr, Karthick; Bender, Florian; Josse, Fabien PhD; Yaz, Edwin E.; and Ricco, Antonio J., "Obtaining Chemical Selectivity from a Single, Nonselective Sensing Film: Two-Stage Adaptive Estimation Scheme with Multiparameter Measurement to Quantify Mixture Components and Interferents" (2018). Electrical and Computer Engineering Faculty Research and Publications. 596.

https://epublications.marquette.edu/electric_fac/596 
Marquette University

e-Publications@Marquette

\section{Electrical and Computer Engineering Faculty Research and Publications/College of Engineering}

This paper is NOT THE PUBLISHED VERSION; but the author's final, peer-reviewed manuscript. The published version may be accessed by following the link in the citation below.

ACS Sensors, Vol. 3, No. 9 (August 17, 2018): 1656-1665. DOI. This article is ( Society and permission has been granted for this version to appear in e-Publications@Marquette. American Chemical Society does not grant permission for this article to be further copied/distributed or hosted elsewhere without the express permission from American Chemical Society.

\section{Obtaining Chemical Selectivity from a Single, Nonselective Sensing Film: Two-Stage Adaptive Estimation Scheme with Multiparameter Measurement to Quantify Mixture Components and Interferents}

Karthick Sothivelr

Department of Electrical and Computer Engineering, Marquette University, Milwaukee, WI

Florian Bender

Department of Electrical and Computer Engineering, Marquette University, Milwaukee, WI

Fabien Josse

Department of Electrical and Computer Engineering, Marquette University, Milwaukee, WI

Edwin E. Yaz

Department of Electrical and Computer Engineering, Marquette University, Milwaukee, WI

Antonio J. Ricco

Department of Electrical Engineering, Center for Integrated Systems, Stanford University, Stanford, CA 


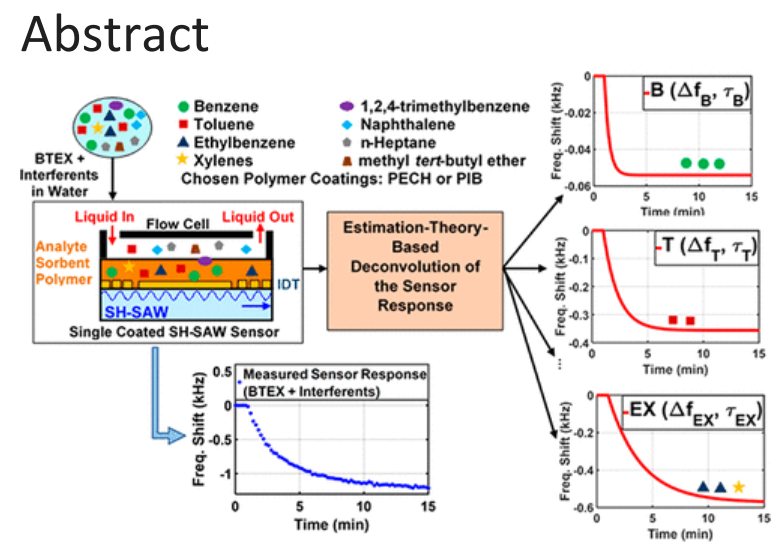

A new approach is reported to detect and quantify the members of a group of small-aromatic-molecule target analytes: benzene, toluene, ethylbenzene, and xylenes (BTEX), dissolved in water, in the presence of interferents, using only the data collected from a single polymer-coated SH-SAW (shear horizontal surface acoustic wave) device and a two-stage adaptive estimation scheme. This technique is composed of exponentially weighted recursive least-squares estimation (EW-RLSE) and a bank of Kalman filters (BKFs) and does not require any prior knowledge of the initial concentration range of the target analytes. The proposed approach utilizes the transient sensor response to sorption and/or desorption of the analytes as well as the error range associated with the response time constants to provide more information about the analyte-specific interactions with the polymer film. The approach assumes that the sensor response to contaminated groundwater is a linear combination of the responses to the single target analytes, the interferents that interact with the selected polymer sensor coatings, and measurement noise. The proposed technique was tested using actual sensor responses to contaminated groundwater samples containing multiple BTEX compounds with concentrations ranging from 10 to 2000 parts per billion, as well as common interferents including ethanol, 1,2,4trimethylbenzene, naphthalene, $n$-heptane, and MTBE (methyl tert-butyl ether). Estimated concentration values, accurate to $\pm 10 \%$ for benzene/toluene and $\pm 15 \%$ for ethylbenzene/xylenes, are obtained in near-real time. The utilization of sorption and/or desorption data enables detection and quantification of BTEX compounds with improved accuracy, high tolerance to measurement noise, and improved chemical selectivity.

\section{KEYWORDS:}

deconvolution of sensor response, chemical sensor selectivity, adaptive estimation, SH-SAW sensors, sensor signal processing

The current applicability of many chemical sensors is limited due to the lack of adequate selectivity to enable real-world applications. In many chemical sensing applications, the chemically sensitive element of the sensor is only partially selective to any specific target analyte, potentially giving rise to errors in analyte identification and quantification. $(1,2)$ Lack of adequate selectivity further increases the challenge in identifying and quantifying target analytes in a mixture. This is often due to the chemical similarity within a group of target analytes, as well as the presence of nontarget interferents. In such cases, enhanced selectivity can be achieved by using an array of appropriately chosen partially selective chemical sensors.(1,2) All sensors in the array respond to most if not all analytes, but the pattern of responses provides a unique fingerprint for each single target species or target mixture. This approach, however, often works well only for single compounds or predefined mixtures. Additional drawbacks of using a sensor array include the challenge of identifying and developing the processing method for each of several different chemically sensitive interfaces, as well as increased signal- and data-processing time and potential misclassification. Data dimensionality and complexity also increase with the number of sensors in the array. If the chemical diversity and/or partial selectivity of the sensor coatings are inadequate, the 
probability of misclassification will increase. In many cases, only one sensing parameter is used per sensor for analyte classification, which may worsen the problem.(2) Using multiple sensor response parameters for classification will improve the selectivity and also help to reduce the number of sensors needed for a sensor array; it may even be possible to use a single sensor device.(3) More reliable sensor signal processing also diminishes error due to misclassification.

One such real-world application which demands selective chemical sensors involves in situ monitoring of groundwater near underground storage tanks (USTs), pipelines, petrochemical processing facilities, and military sites for accidental releases of petroleum hydrocarbons. The groundwater near USTs is subject to legal monitoring requirements for accidental releases of fuel and oil.(4) Contaminated groundwater samples often contain many chemically similar organic compounds, complicating target detection and quantification. In this application, the volatile, low-molecular-weight organic compounds (VOCs) benzene, toluene, ethylbenzene, and xylenes (BTEX) are the target analytes of interest because they can be found in crude oil and its refined products, and are therefore used as indicators of gasoline releases. $(5,6)$ The presence of BTEX compounds in groundwater is a major concern due to their hazard potential and relatively high solubility in water.(6-9) Specifically, detection of benzene in water is of importance due to its carcinogenicity. $(3,9,10)$ Maximum permitted contaminant levels of BTEX compounds in drinking water are in the low ppb $(\mu \mathrm{g} / \mathrm{L})$ to low ppm $(\mathrm{mg} / \mathrm{L})$ range.(8) Thus, it is imperative to monitor the presence of BTEX compounds in groundwater at critical sites regularly so that remediation actions can be carried out swiftly if leaks are detected. The detection and quantification of BTEX analytes in groundwater is challenging due to the chemical similarity of the BTEX compounds to one another and the presence of other nontarget interferents such as dissolved salts, aliphatic hydrocarbons, dissolved gases, and so forth.

Currently, USTs are only monitored in two-to-three-year intervals due to the high cost and labor-intensive process involved in sample collection at the monitoring well and shipping to an ex situ laboratory for manual analysis.(11) Because of the long monitoring intervals, leakage can go unnoticed for long periods of time. Therefore, for rapid assessment of the contamination of groundwater and surface water with petroleum products, an in situ sensor system with high sensitivity to dissolved BTEX compounds is needed, especially for sites that include multiple interferents; such a system should be capable of more accurate, inexpensive, and near-real-time long-term monitoring of groundwater.

The conventional techniques used to detect and quantify BTEX compounds involve either spectroscopy or gas chromatography.(12,13) Examples include infrared evanescent field spectroscopy $(12-14)$ and Raman spectroscopy.(15) Estimation of BTEX in groundwater using a combination of gas chromatography and mass spectrometry was also reported.(16) Although these methods are capable of accurately quantifying BTEX compounds, there are several drawbacks associated with these techniques. For instance, while Raman spectroscopy $(15)$ can be made field-deployable, its portable version lacks the necessary detection limits for BTEX compounds (i.e., BTEX detection limits are relatively high on the order of several ppm). Other techniques such as infrared evanescent field spectroscopy $(12-14)$ are relatively impractical for use as a field-deployed system due to their total size and complexity in sample preparation procedures.(12)

Chemical sensor arrays based on various sensing platform technologies such as optical fibers, chemiresistors, and SAW (surface acoustic wave) sensors have been investigated for the detection and quantification of BTEX compounds in water and soil.(17-22) The detection based on sensor arrays usually requires the use of lineardiscriminant analysis, principal-component analysis, $k$-nearest-neighbor algorithms or other methods of pattern recognition.

Previously, we reported a sensor system for the detection and quantification of BTEX compounds that makes use of a polymer-coated shear-horizontal surface acoustic wave (SH-SAW) device and a signal-processing 
technique based on a bank of extended Kalman filters (EKFs).(23) To extend the applicability of that approach, a more systematic treatment of the contribution of interferents in the model of the sensor response is still needed to achieve more accurate detection and estimation of analyte concentrations. For a given set of environments of interest, it is critical to account for all probable interferents to which the selected polymer coating responds. In addition, rigorous tests on the sensor system reveal several drawbacks with the signal processing technique based on a bank of EKFs. The EKF is not an optimal filter in this context because it is based on linearization of a nonlinear model of the system. Thus, the convergence of EKF analysis depends on the initial value of the state estimate, the error covariance, and the values of process and measurement noise.(24) Therefore, this approach only works well if the approximate initial concentration range of the BTEX analytes is known a priori.

In this work, we present a new approach for the detection and quantification of $n$ analytes (here, the six BTEX compounds, including the three isomers of xylene) in aqueous liquids and in the presence of interferents using only the data collected from a single polymer-coated SH-SAW device and an estimation-theory-based technique comprising an exponentially weighted recursive least-squares estimation (EW-RLSE) and a bank of Kalman filters (BKFs). This approach is based on a linear sensor response model and does not require any prior knowledge of the initial concentration range of the analytes in the sample in order to accurately estimate their concentrations. The linear sensor response model is formulated by assuming that the response of a single polymer-coated $\mathrm{SH}$ SAW device to contaminated groundwater is a linear combination of the responses to the single target analytes, the interferents that interact with the selected polymer coatings, and measurement noise. For a number of contaminated groundwater samples collected in the field, it has been found empirically that the response to 1,2,4-trimethylbenzene dominates the response due to the interferents for the selected coating. Thus, the contribution of this interferent (as well as other analytes) to the sensor response is treated individually as one analyte, provided that the sensitivity and response time are non-negligible. The ultimate purpose of the sensor signal-processing technique is to facilitate the detection and accurate quantification of the target analytes in the presence of noise and interferents. By using both sorption and desorption transient response data, obtained as the flow across the sensor is switched from pure water to the analyte stream, and subsequently back again to pure water, more information is provided about the analyte-specific interactions with the polymer film. This enables detection and quantification of target analytes with improved accuracy, high tolerance to measurement noise, and improved selectivity. Using extensive experimental data from various mixture compositions, compared to previous work,(23) the proposed approach produces more accurate concentration estimates with greater reliability, high tolerance to measurement noise, improved chemical selectivity, and near-real-time data processing.

\section{Modeling the Sensor Response}

The use of estimation-theory-based technique to analyze sensor data for the detection and quantification of target analytes in the presence of interferents requires a relatively accurate analytical model that describes the response of the SH-SAW sensor to the response transients generated by exposure to samples. Based on empirical data, a general multianalyte sensor response model was developed. The general multianalyte model was then adapted for the detection and quantification of target analytes in the presence of interferents.

\section{A. General Model of the Multiple-Analyte Sensor Response}

For the sensor response to multiple analyte samples, two important assumptions were made. First, it was assumed that sorption of the mixture by the polymer obeys Henry's law, namely, that the concentration of the various mixture components in the coating at any time $t$ is the sum of the concentrations of the individual analytes $\left(C_{i}(t)\right)$ that would be measured in single-analyte responses. Free partitioning of analytes between polymer and aqueous phase is inferred, also implying that the sorption process is reversible at room temperature and that only physisorption occurs. For BTEX compounds, based on experimental observations, it is 
found that Henry's law is valid for analyte concentrations in the range of 0 to $50 \mathrm{ppm}(\mathrm{mg} / \mathrm{L})$ depending on the sorbent coating.(6) The process of analyte sorption/desorption can then be assumed to follow first-order kinetics (similar to the model of single-analyte sensor response(23)). Second, Henry's law behavior along with comparatively low analyte concentrations dissolved in the polymer film ensure that, at any time $t$, the change in frequency due to the mixture is the sum of the frequency changes due to each analyte in the mixture. Based on these two assumptions, the sensor response to a mixture of $n$ analytes can be written as

$\Delta f(t)=-\sum_{i=1}^{n} a_{i} C_{i}(t)(1 \mathrm{a})$

where $\Delta f(t)$ is the observed frequency shift at time $t$, the subscript $i=1,2, \cdots, n$ refers to each analyte in the mixture, $a_{i}$ is a sensitivity parameter for each analyte (which is a function of the sensor platform, the sensor coating, and the analyte(25)), and $C_{i}(t)$ is the concentration of each analyte in the coating at time $t$. $C_{i}(t)$ is the solution to the following first-order differential equation

$\dot{C}_{i}(t)=-\frac{1}{\tau_{i}} C_{i}(t)+\frac{K_{p-w, i}}{\tau_{i}} C_{a m b, i}(t)$

where $C_{\mathrm{amb}, i}(t)$ is the ambient concentration of each analyte at time $t$ (which for these experiments remains constant due to the constant flow of fresh analyte-containing sample solution throughout the measurement), $\tau_{i}$ is the response time constant of each analyte for a given coating, and $K_{\mathrm{p}-\mathrm{w}, i}$ is the polymer/water partition coefficient of each analyte for a given coating.(25) Equation 1 represents the general analytical model for the sensor response to any number of analytes in the sample, provided that each possible target analyte and all interferents in the sample have been separately characterized for the appropriately selected coating in the relevant concentration range.

For the desorption process (which is measured by exposing the sensor to clean (or filtered) water after the sorption process reaches steady state), it is also observed that the sensor signal changes rapidly at first and then more slowly as it approaches steady state. Thus, the desorption process can also be described using eq 1 . The difference between absorption and desorption is reflected in the sign of the frequency shift, $\Delta f(t)$. For the investigated sensors and coatings, the sorption process produces a negative frequency shift and desorption a positive frequency shift. It should be noted that, based on single-analyte experiments for a given analyte, sorption and desorption responses display the same absolute magnitude of equilibrium frequency shiftmeaning the responses are reversible, but they do not necessarily have the same response-time constants. Empirically, it is observed that desorption time constants are slightly different from sorption time constants, but the difference is usually within the experimental error margins. The mechanistic implication of this observation is that the dominant factor in determining the time constants is the rate of diffusion of each analyte through the polymer coating.

To implement the estimation-theory-based technique, the model represented by eq 1 has to be converted into state-space form. For that purpose, eq 1 is normalized and discretized first. This is because the sensor response data are collected at discrete times. The model is normalized by dividing eq 1 by $K_{\mathrm{p}-\mathrm{w}, i} C_{\max , i}$ (where $C_{\max , i}$ represents the equilibrium ambient concentration). By introducing new variables,

$m_{i}(t)=\frac{C_{i}(t)}{K_{\mathrm{p}-\mathrm{w}, \mathrm{i}} C_{\mathrm{max}, \mathrm{i}}}(2 \mathrm{a})$
$u_{i}(t)=\frac{C_{\mathrm{amb}, \mathrm{i}}(t)}{C_{\mathrm{max}, \mathrm{i}}}(2 \mathrm{~b})$

and

$\alpha_{i}=-\alpha_{i} K_{\mathrm{pw}, \mathrm{i}} C_{\max , \mathrm{i}}(2 \mathrm{c})$ 
the following normalized equations are obtained

$\dot{m}_{i}(t)=-\frac{1}{\tau_{i}} m_{i}(t)+\frac{1}{\tau_{i}} u_{i}(t)$

and

$\Delta f(t)=\sum_{i=1}^{n} \alpha_{i} m_{i}(t)(3 \mathrm{~b})$

where, for analyte $i, m_{i}(t)$ is the normalized concentration absorbed/desorbed at time $t, \alpha_{i}$ is the equilibrium frequency shift, and $u_{i}(t)$ is the concentration profile to which the sensor responds during switching from clean water to the sample, or vice versa for desorption. Mathematically, $u_{i}(t)$ can be described using a modified error function

$u_{i}(t)=\frac{1}{2}\left[1-\operatorname{erf}\left(\frac{1-v t}{2 \sqrt{D t}}\right)\right](4)$

where $/$ is the total length of the tube separating the point of sample introduction and the sensor device, $v$ is the average flow speed, and $D$ is the diffusion coefficient of the soluble substance. $(26,27)$ For the measurement system used to collect the data analyzed in this paper, $u_{i}(t)$ can be approximated by a unit step function (for $t<$ $0, C_{a m b, i}(t)=0$; for $t>0, C_{a m b, i}(t)=C_{\max }$, or vice versa for desorption) provided the initial few data points (depending on the data collection sampling period) are ignored.

Since the sensor response data are collected at discrete times (i.e., $t=k T_{\mathrm{s}}$, where $T_{\mathrm{s}}$ is the sampling period and $k \geq 0$ is an integer), the continuous-time normalized model of eq 3 is converted into a discrete-time model using Euler's first-order forward method

$m_{i, k+1}=\left(1-S_{i}\right) m_{i, k}+S_{i} u_{i, k}(5 a)$

and

$\Delta f_{k}=\sum_{i=1}^{n} \alpha_{i} m_{i, k}+w_{k}(5 \mathrm{~b})$

where $S_{i}$ is the sorption/desorption rate constant (i.e., $S_{i}=\frac{T_{i}}{\tau_{i}}$ ). The term $w_{k}$ represents the measurement noise with variance $\sigma_{w}{ }^{2}$, which likely is present during data collection. It is assumed that the measurement noise is white noise (uncorrelated in time).

From eq 5 , the state-space form can be obtained by assigning state variables to the normalized concentrations absorbed/desorbed at time $t, m_{i, k}$

$y_{k}=\Delta f_{k}=\sum_{i=1}^{n} a_{i} x_{k}^{(i)}+w_{k}=C^{T} x_{k}+w_{k}(6 \mathrm{a})$

and

$\left[\begin{array}{c}x_{k+1}^{(1)} \\ x_{k+1}^{(2)} \\ \vdots \\ x_{k+1}^{(n)}\end{array}\right]=\left[\begin{array}{cccc}1-S_{i} & 0 & 0 & 0 \\ 0 & 1-S_{2} 0 & 0 \\ 0 & 0 & \ddots & 0 \\ 0 & 0 & 0 & -S_{n}\end{array}\right]\left[\begin{array}{c}x_{k}^{(1)} \\ x_{k}^{(2)} \\ \vdots \\ x_{k}^{(n)}\end{array}\right]+\left[\begin{array}{c}S_{1} \\ S_{2} \\ \vdots \\ S_{n}\end{array}\right] U_{k}=A x_{k}+B U_{k}$

where

$C^{T}=\left[\alpha_{1} \alpha_{2} \ldots \alpha_{n}\right]$ 
$A=\left[\begin{array}{cccc}1-S_{i} & 0 & 0 & 0 \\ 0 & 1-S_{2} 0 & 0 \\ 0 & 0 & \ddots & 0 \\ 0 & 0 & 0 & -S_{n}\end{array}\right]$

$B=\left[\begin{array}{llll}S_{1} S_{2} & \cdots & S_{n}\end{array}\right]^{T}$

$x_{k}=\left[\begin{array}{llll}x_{k}^{(1)} & x_{k}^{(2)} & \cdots & x_{k}^{(n)}\end{array}\right]^{T}$

In eq $6, x_{k}^{(i)}$ represents the normalized concentration of absorbed/desorbed analyte at time $t, A$ and $B$ represent the state matrices, and $C$ is a vector of the equilibrium (or steady-state) frequency shifts, $\alpha_{i}$, which are the parameters to be estimated. Using the state-space form of eq 6 and estimation-theory-based technique, the $\alpha_{i}$ value for each analyte can be estimated in near-real time using the measured total frequency shift, $\Delta f_{k}$.

\section{B. Model of the Target Analyte Sensor Response in the Presence of Interferents}

For the quantitative analysis of the target analytes in the presence of interferents, it is important to identify all the interferents that respond to the selected polymer coatings. Often, detectable interferents will either have longer response times or lower sensitivities than the target analytes. Based on the sensor response of the detectable interferents, several different representations of the target analytes in the presence of interferents are possible using eq 6 :

Case 1: If all the detectable interferents have distinct sensor parameters (i.e., response time constants and sensitivities) each interferent must be explicitly represented using an individual exponential term. In this case, eq 6 can be used to represent the response due to the $p$ detectable interferents using $p$ individual exponential terms and $z$ target analytes using $z$ exponential terms where $n=z+p$.

Case 2: If all the detectable interferents have very low sensitivity for detection using the chosen polymer coatings (low sensitivity in the sense that the resulting signals for these interferents will always be much less than any signal for any target analyte), the response due to the interferents can be ignored. In this case, eq 6 can be used alone to represent the response due to the target analytes.

Case 3: If the responses due to all the detectable interferents have similar time constants and sensitivities, the combined response due to the interferents can be represented using one single-analyte term. In this case, eq 6 can be used to represent the response due to all detectable interferents using one individual exponential term (i.e., $p=1$ ) and $z$ target analytes using $z$ exponential terms where $n=z+1$.

Case 4: If the responses due to multiple groups of similarly responsive interferents have different time constants (and sensitivities if separate quantitation is needed), the combined response due to each group of interferents with similar sensor parameters can be represented using one exponential term. In this case, eq 6 can be used to represent the response due to each group of detectable interferents using one individual exponential term (i.e., $p=p_{1}+p_{2}+p_{3}+\ldots$ ) and $z$ target analytes using $z$ exponential terms where $n=z+p$.

In this paper, the multianalyte model is used to treat the detection and quantification of BTEX compounds in water in the presence of interferents. In order to utilize the multianalyte model, each target BTEX compound and each possible interferent (in its concentration range of interest) in the sample must be separately characterized for the selected polymer coatings, with the following considerations particular to this application scenario:

1. Target Analytes - The average values of response time constants and sensitivities for the target BTEX analytes extracted from the sorption and sorption responses for the investigated coatings are listed in Tables 1 and $\underline{2}$. As chemical isomers, for the investigated sensor coatings, ethylbenzene and the mixture of $m-, o_{-}$, and $p$-xylenes 
are found to have nearly identical values for the response times and sensitivities; thus, no attempt was made to distinguish between them.

Table 1. Measured Average Sensitivities and Response Times for $0.6 \mu \mathrm{m}$ PECH-Coated SH-SAW Sensor to Various BTEX Analytes and Common Interferents ${ }^{\mathbf{a}}$

\begin{tabular}{|c|c|c|c|c|}
\hline & Mean Sensitivity, $a K_{\mathrm{p}-\mathrm{w}}(\mathrm{Hz} / \mathrm{ppm})$ & & Mean Response Time, $\tau(s)$ & \\
\hline Analyte & Sorption & Desorption & Sorption & Desorption \\
\hline Benzene & $109( \pm 9)$ & $110( \pm 11)$ & $27( \pm 8)$ & $27( \pm 9)$ \\
\hline Toluene & $435( \pm 25)$ & $422( \pm 4)$ & $78( \pm 3)$ & $74( \pm 5)$ \\
\hline Ethylbenzene & $1450( \pm 240)$ & $1410( \pm 240)$ & $175( \pm 13)$ & $171( \pm 13)$ \\
\hline 1,2,4-Trimethylbenzene & $3540( \pm 420)$ & $3390( \pm 410)$ & $428( \pm 22)$ & $461( \pm 27)$ \\
\hline Naphthalene & $1560( \pm 30)$ & $1605( \pm 27)$ & $495( \pm 87)$ & $665( \pm 24)$ \\
\hline n-Heptane & $\approx 0$ & $\approx 0$ & N/A & N/A \\
\hline Ethanol & $\approx 0$ & $\approx 0$ & N/A & N/A \\
\hline MTBE & 《benzene & «benzene & <benzene & <benzene \\
\hline
\end{tabular}

${ }^{\mathrm{a} N} / \mathrm{A}=$ not applicable; $\mathrm{PECH}=$ poly(epichlorohydrin) .

Table 2. Measured Average Sensitivities and Response Times for $0.8 \mu \mathrm{m}$ PIB-Coated SH-SAW Sensor to Various BTEX Analytes and Common Interferents ${ }^{\mathbf{a}}$

\begin{tabular}{|c|c|c|c|c|}
\hline & Mean Sensitivity, $a K_{\mathrm{p}-\mathrm{w}}(\mathrm{Hz} / \mathrm{ppm})$ & & Mean Response Time, $\tau(\mathrm{s})$ & \\
\hline Analyte & Sorption & Desorption & Sorption & Desorption \\
\hline Benzene & $78( \pm 7)$ & $78( \pm 12)$ & $36( \pm 7)$ & $31( \pm 3)$ \\
\hline Toluene & $403( \pm 39)$ & $408( \pm 85)$ & $88( \pm 7)$ & $88( \pm 9)$ \\
\hline Ethylbenzene & $1160( \pm 57)$ & $1100( \pm 85)$ & $230( \pm 12)$ & 215 ( \pm 11$)$ \\
\hline 1,2,4-Trimethylbenzene & $3640( \pm 230)$ & $3440( \pm 165)$ & $610( \pm 18)$ & $667( \pm 31)$ \\
\hline Naphthalene & 621 & 650 & 250 & 254 \\
\hline$n$-Heptane & 》ethylbenzene & 5930 & 》ethylbenzene & 9180 \\
\hline Ethanol & $\approx 0$ & $\approx 0$ & N/A & N/A \\
\hline MTBE & «benzene & «benzene & <benzene & <benzene \\
\hline
\end{tabular}

${ }^{\mathrm{a}} \mathrm{N} / \mathrm{A}=$ not applicable; $\mathrm{PIB}=$ poly(isobutylene).

2. Interferents - The coated sensors were exposed to some interferents commonly found in contaminated groundwater, namely, ethanol, 1,2,4-trimethylbenzene, naphthalene, $n$-heptane, and MTBE (methyl tert-butyl ether). For concentrations up to 100 ppm, no significant sensor response to ethanol was observed. For MTBE, a very low sensitivity was found for the selected coatings ( $1 \mathrm{~Hz} / \mathrm{ppm})$ and, since this compound is usually present at low concentrations in groundwater, its contributions to the sensor responses were neglected. For 1,2,4trimethylbenzene and naphthalene, high sensitivity and long response times were observed, indicating that the response to these compounds cannot be ignored. For poly(isobutylene) (PIB) sensor coatings, non-negligible responses to $n$-heptane were also found. Therefore, the characteristic response time constants and sensitivities of 1,2,4-trimethylbenzene, naphthalene and $n$-heptane were extracted from the sensor responses of the investigated coatings and are listed in Tables 1 and $\underline{2}$. Due to its lower solubility in water, the concentration of $n$ heptane is usually much lower than that of 1,2,4-trimethylbenzene. Based on the analysis performed on these common interferents, it has been found that the response to 1,2,4-trimethylbenzene dominates the contribution of the interferents to the sensor response. All the above information is used in devising the models for the detection and quantification of BTEX compounds in the presence of interferents.

Based on the characterization of each target analyte (the BTEX compounds as defined above) and possible interferent, a five-analyte model $(n=5)$ was selected for the detection and quantification of BTEX in the presence of interferents. In this model, the first three exponential terms $(i=1,2,3)$ represent the response due to the BTEX compounds and the last two exponential terms $(i=4,5)$ represent the response due to all 
detectable interferents in the mixture with a response time constant longer than that of all BTEX analytes. The choice to represent the overall interferent response by only two exponential terms is based on empirical data showing that the response to 1,2,4-trimethylbenzene dominates the interferent response. Therefore, 1,2,4trimethylbenzene is treated individually using one exponential term $(i=4)$ and the other exponential term $(i=5)$ represents the contributions of other less important interferents. It is noted that the time constant for $i=5$ is set close to the characteristic time constant of C10 aromatic compounds, consistent with the fact that besides 1,2,4trimethylbenzene and similar C9 aromatic compounds, the second largest group of interferents identified in the test samples are $\mathrm{C} 10$ aromatics, which are expected to show even longer response times. The state-space form of the five-analyte model is as follows:

$y_{k}=\Delta f_{k}=\sum_{i=1}^{5} \alpha_{i} x_{k}^{(i)}+w_{k}(7 a)$

And

$$
\left[\begin{array}{l}
x_{k+1}^{(1)} \\
x_{k+1}^{(2)} \\
x_{k+1}^{(3)} \\
x_{k+1}^{(4)} \\
x_{k+1}^{(5)}
\end{array}\right]=\left[\begin{array}{ccccc}
1-S_{1} & 0 & 0 & 0 & 0 \\
0 & 1-S_{2} & 0 & 0 & 0 \\
0 & 0 & 1-S_{3} & 0 & 0 \\
0 & 0 & 0 & 1-S_{4} & 0 \\
0 & 0 & 0 & 0 & 1-S_{5}
\end{array}\right]\left[\begin{array}{l}
x_{k}^{(1)} \\
x_{k}^{(2)} \\
x_{k}^{(3)} \\
x_{k}^{(4)} \\
x_{k}^{(5)}
\end{array}\right]\left[\begin{array}{l}
(7 \mathrm{~b}) \\
S_{1} \\
S_{2} \\
S_{3} \\
S_{4} \\
S_{5}
\end{array}\right]
$$

\section{Sensor Signal Processing}

The purpose of sensor signal processing is to facilitate the detection and accurate quantification of the target analytes in the presence of noise and interferents. In this work, an estimation-theory-based technique is used for sensor response data processing because it offers various advantages, including its ability to accurately estimate the states even in the presence of noise (both measurement and process noise), near-real-time data processing, and minimal computational and memory requirements for real-world implementations. Since the state-space form is "linear in the unknown parameters" (they occur linearly in eq 7a), linear estimation theory is selected. In particular, based on our investigations, an estimation-theory-based technique comprising EW-RLSE and BKFs (two-step processing) was selected as being most suitable. A brief review of EW-RLSE and BKFs is given in the Supporting Information.

The sensor signal-processing procedure used to obtain the final estimated concentrations of BTEX compounds in the presence of interferents using the data collected from only a single polymer-coated SH-SAW device is summarized in Figure 1. Both sorption and desorption data are utilized. Note that the error range in the measured mean response times for sorption and desorption as shown in $\underline{\text { Tables} 1}$ and $\underline{2}$ were taken into account in the signal-processing procedure. Incorporating desorption transients (which can be more sensitive to energies of desorption of analyte targets, particularly for comparatively larger energies of interaction) in the signalprocessing procedure provides more information about the analyte/polymer interactions that can be used to obtain more accurate estimated concentrations for BTEX compounds compared to using sorption transients alone. Moreover, utilizing sorption and desorption data in the signal processing reduces the error in the estimation due to measurement noise, making this procedure highly tolerant of such noise. In short, this approach improves the accuracy of the extracted concentrations and the reliability of chemical speciation. 


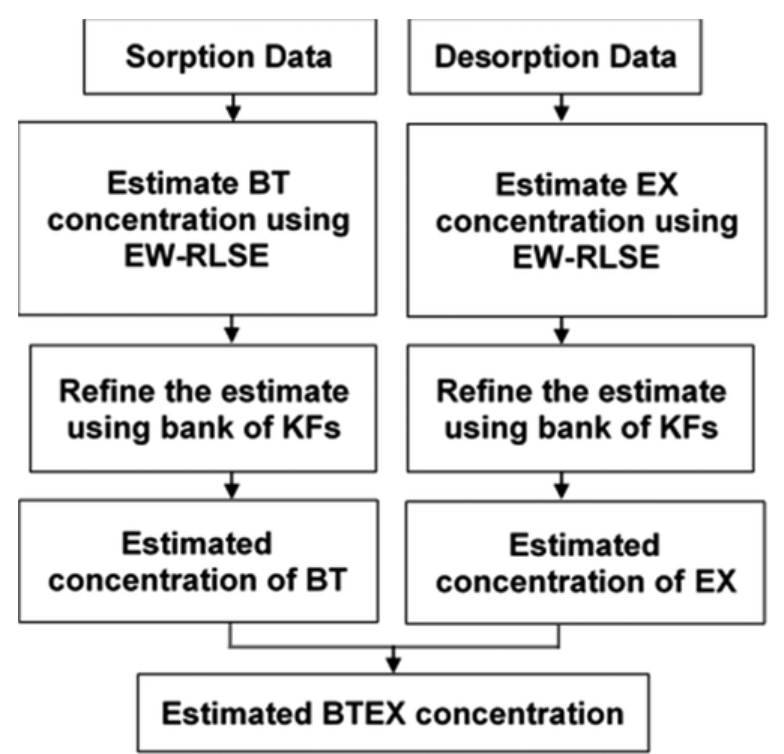

Figure 1. Estimation-theory-based sensor signal processing procedure that utilizes sorption and desorption data for detection and quantification of BTEX analytes. "BT" and "EX" refer to the respective BTEX analytes.

Based on our tests, it is observed consistently that using the sorption data results in more accurate estimates of benzene and toluene concentrations whereas using the desorption data results in more accurate estimates for the combined concentrations of ethylbenzene and xylenes. We believe this is due to the analytes' general interaction with (and diffusion through) the polymer coatings, i.e., to minor deviations from strict Henry's law behavior. During analyte sorption, compounds with shorter response times ( $B$ and $T$ ) are absorbed into a relatively "empty" coating, similar to the case of single-analyte detection that was used to determine individual response time constants and sensitivities. The remaining compounds (EX, interferents) are mostly absorbed into a coating that already contains $B$ and $T$, thus introducing a slight error-some change in their rates of diffusion due to the presence of $B$ and $T$. The reverse is found to be true for analyte desorption: much of the desorption of EX occurs after B,T have already vacated the film, but the diffusion of $B$ and T may be slightly influenced by the presence in the film of EX. Therefore, in the proposed signal-processing procedure, sorption data are used to estimate $B$ and $T$ concentrations whereas desorption data are used to obtain an estimate for the combined concentrations of EX. For sorption and/or desorption, the algorithm obtains the estimates using two-step processing (EW-RLSE and BKFs). The bank is formed as a parallel combination of KFs, each designed for a different set of frequency shifts, $\alpha_{i}$, with the range of $\alpha_{i}$ based on the frequency shifts associated with the extracted concentrations from EW-RLSE. The frequency shifts, $\alpha_{i}$ from EW-RLSE, are increased and decreased by $20 \%$ to define limits for a range of possible equilibrium frequency shifts for the analytes. Within these limits, a finite number of $\alpha_{i}$ is obtained by uniformly quantizing the equilibrium frequency shifts which are used to form the vector $C$ in eq 6 . Note that, for this technique, no initial values of the unknowns are needed; therefore, any concentration range of BTEX compounds can be analyzed and the results can be obtained in near real-time. The proposed signal-processing procedure is tested and validated using experimental data.

\section{Chemical Sensor Data Acquisition}

In order to test and validate the performance of the proposed technique, experimental sensor data were

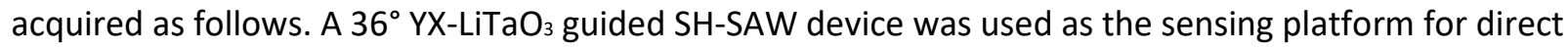
measurement in aqueous phase as described previously. $(23,34)$ The operating frequency of the polymer-coated sensor devices have a frequency of operation of $103 \mathrm{MHz}$. To eliminate the effects of environmental parameters such as temperature and pressure variations on the sensor response, a dual-delay-line configuration was used with one line as the sensing line and the other as the reference line. $(23,34)$ The sensing area of both delay lines 
are metalized to reduce/eliminate acoustoelectric interactions with the load (i.e., the liquid under investigation). The sensing line is coated with the chemically sorbent polymer coatings, either poly(epichlorohydrin) (SigmaAldrich, St. Louis, MO) or poly(isobutylene) (Sigma-Aldrich). These polymers were dissolved in chloroform, spin coated onto the sensing line, and baked for $15 \mathrm{~min}$ at $55^{\circ} \mathrm{C}$, resulting in thicknesses of 0.6 and $0.8 \mu \mathrm{m}$ for PECH and PIB, respectively. The baking step (in effect, annealing the polymer film) is crucial to ensure repeatability of the sensor responses. Poly(methyl methacrylate) (PMMA) (Scientific Polymer Products, Ontario, NY) coating, baked for $120 \mathrm{~min}$ at $180^{\circ} \mathrm{C}$, resulting in a relatively glassy nonsorbent layer was used for the reference line as the chemically insensitive material for the concentration range of interest. The BTEX analytes used in the experiments had purities of at least $98.5 \%$ and were purchased from Sigma-Aldrich. BTEX analytes were dissolved at the desired concentrations in deionized (DI) water or groundwater. Groundwater and light nonaqueous-phase liquid (LNAPL) samples used in the experiments were collected from actual monitoring wells in California. The concentrations of these samples are given in parts per million or parts per billion by weight. Some interferents commonly found in groundwater were also tested. These include $n$-heptane, 1,2,4trimethylbenzene, naphthalene, MTBE, and ethanol. All the above listed interferents were obtained from SigmaAldrich with purity of at least $98 \%$, except for ethanol that was denatured and had purity of at least $90 \%$.

The sensor data was collected as described in our previous publications. $(23,34)$ An independent measurement of BTEX concentrations was obtained for the aqueous LNAPL solutions using a portable GC-PID (gas chromatograph-photoionization detector) system (Defiant Technologies FROG-4000, Albuquerque, NM). When needed, analyte concentrations were further verified using GC-MS (gas chromatography-mass spectroscopy). The LNAPL samples were prepared as described previously $(23,34)$ to yield concentrations of $\leq 1 \mathrm{ppm}$ for each BTEX compound. To minimize the loss of volatile analytes, the headspace in the sample vials was kept negligible. For the experiments, the coated sensor device was placed inside a water flow cell designed in-house;(35) a peristaltic pump (IDEX Ismatec Reglo Digital MS, Oak Harbor, WA) was used for the sample flow. To minimize hydrodynamic forces from the flowing fluid, the solutions were pumped at a constant flow rate of $7 \mu \mathrm{L} / \mathrm{s}$. A stable baseline output signal was obtained by drawing in a reference solution (DI water or groundwater) before introducing the analyte(s) into the flow cell. Filtration of the groundwater samples for removal of sediments and other physical interferents was also performed before usage. Once the sensor response to the analyte reaches equilibrium, the flow cell was flushed again with the reference solution, causing the analyte(s) to desorb from the sensor coating. The process was repeated for different analyte samples and concentrations. All measurements were conducted at room temperature $\left(22 \pm 0.05^{\circ} \mathrm{C}\right)$.

\section{Results and Discussion}

This section presents the results on detection and quantification of target analytes (BTEX compounds) in the presence of interferents using the proposed signal processing approach and the $n$-analyte model described above. The new approach was tested extensively using measured sensor responses (i.e., frequency shifts) to BTEX compounds in groundwater. Since the proposed technique is independent of the initial values of the unknown parameters (i.e., the equilibrium frequency shifts, $\alpha_{i}$ ), the initial values of all the unknown parameters were set to zero for all the tested measurement data. Once the unknown parameters are estimated, the corresponding concentrations associated with each of these unknown parameters (analytes) are extracted using the measured mean sensitivities of BTEX compounds as listed in Table1 (for PECH) and Table2 (for PIB). The extracted results were then compared to the BTEX concentrations independently obtained from the GC-PID (and GC-MS). It is important to point out that the BTEX concentrations obtained from the GC-PID have an average error of $\pm 7 \%$.(36)

A large number of experiments with BTEX-containing samples were conducted. The estimated concentrations obtained from these tests are summarized in Figures 4 and $\underline{5}$ at the end of this section. Two representative 
estimation results for each coating (i.e., PECH and PIB) are presented first to highlight the effectiveness of the proposed technique. Figure 2 and Table3 show results for sensor response data collected using the sensor device coated with $0.6 \mu \mathrm{m}$ PECH to a LNAPL sample, which contains $390 \mathrm{ppb}$ benzene, $580 \mathrm{ppb}$ toluene, and 75 ppb ethylbenzene and xylenes in the presence of interferents commonly found in groundwater. Figure 2 shows very good agreement between the measured data and the estimated sensor response obtained using sorption and desorption response data. The concentrations for BTEX compounds estimated using the proposed signal processing procedure are summarized in Table3. Those results indicate very good agreement between the concentrations measured using GC-PID and the concentrations estimated using the proposed approach. All estimated concentrations are well within $10 \%$ of the GC-PID measurements.
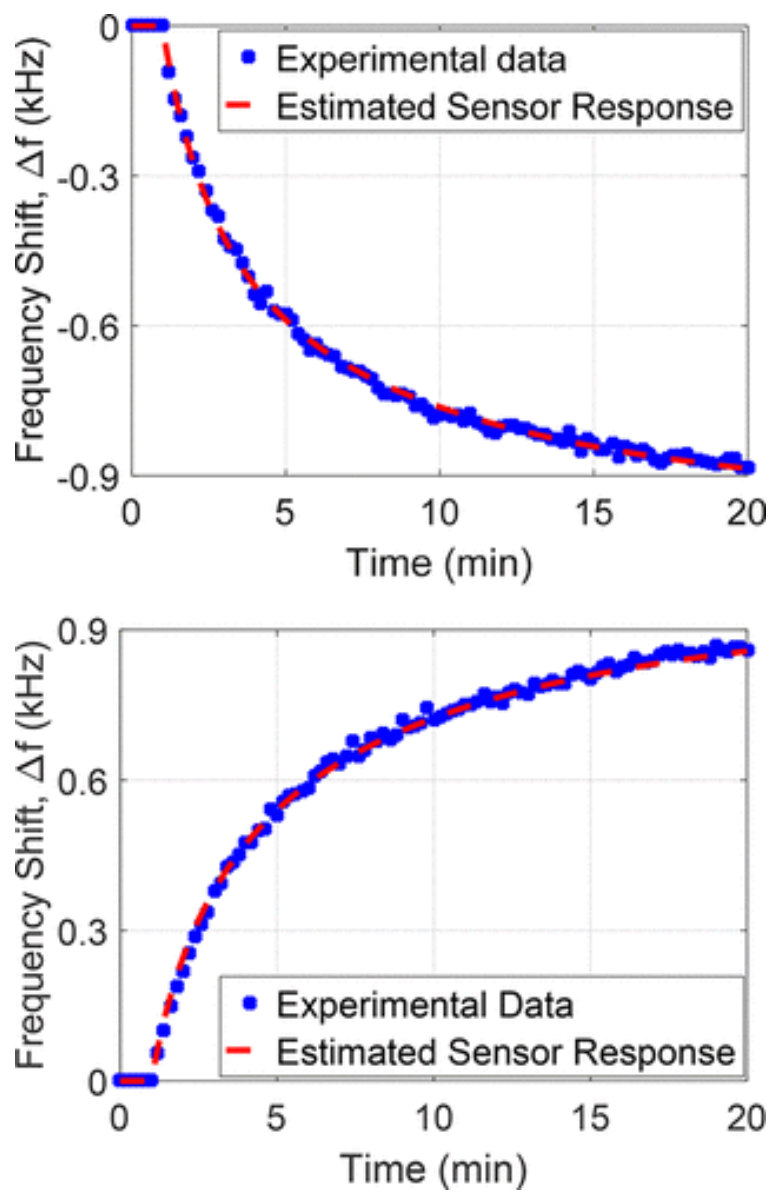

Figure 2. Measured response of a SH-SAW sensor with $0.6 \mu \mathrm{m}$ PECH coating to a solution of LNAPL in groundwater containing $390 \mathrm{ppb}$ benzene, $580 \mathrm{ppb}$ toluene, $75 \mathrm{ppb}$ ethylbenzene/xylenes, and unknown concentrations of interferents (top: sorption data, bottom: desorption data). Also shown (red dashed line) are the estimated sensor responses obtained using the five-analyte model.

Table 3. Estimated Concentrations of BTEX Compounds Obtained using the Measured Response to LNAPL in Groundwater ${ }^{\text {a }}$ Compared to Concentrations Measured using GC-PID

\begin{tabular}{|l|l|l|}
\hline Target Analyte & GC-PID (ppb) & Estimated (ppb) [\% difference] \\
\hline Benzene & $\mathbf{3 9 0}$ & $\mathbf{3 5 3}[\mathbf{9 \%}]$ \\
\hline Toluene & $\mathbf{5 8 0}$ & $\mathbf{5 3 3}[\mathbf{8 \%}]$ \\
\hline Ethylbenzene and Xylenes & $\mathbf{7 5}$ & $\mathbf{7 0}[\mathbf{7 \%}]$ \\
\hline
\end{tabular}

${ }^{\mathrm{a} C}$ Collected using a SH-SAW sensor with $0.6 \mu \mathrm{m} \mathrm{PECH}$ coating. 
The estimation results obtained for the response of a sensor device coated with $0.8 \mu \mathrm{m}$ PIB to a LNAPL sample, which contains $400 \mathrm{ppb}$ benzene, $800 \mathrm{ppb}$ toluene, and $550 \mathrm{ppb}$ ethylbenzene and xylenes in the presence of interferents, are shown in Figure 3 and Table4. Based on Figure 3, it can be observed that the estimated response curve is in close agreement with the measured data which indicate that the estimated equilibrium frequency shifts, $\alpha_{i}$, are indeed close to the actual values. In Table4, the estimated concentrations are in very good agreement with the concentrations determined using GC-PID. In this case, all estimated concentrations are within $15 \%$ of the GC-PID measurements. It is important to note that for the sample results shown, if the desorption data were not used in the proposed signal-processing procedure, the estimation error for ethylbenzene and xylenes would have been much higher. These results clearly illustrate the effectiveness of the proposed technique.

Table 4. Estimated Concentrations of BTEX Compounds Obtained Using the Measured Response to LNAPL in Groundwater- Compared to Concentrations Measured using GC-PID

\begin{tabular}{|l|l|l|}
\hline Target Analyte & GC-PID (ppb) & Estimated (ppb) [\% difference] \\
\hline Benzene & $\mathbf{4 0 0}$ & $\mathbf{4 2 0}[\mathbf{5 \%}]$ \\
\hline Toluene & $\mathbf{8 0 0}$ & $\mathbf{8 9 0}[\mathbf{1 1 \%}]$ \\
\hline Ethylbenzene and Xylenes & $\mathbf{5 5 0}$ & $\mathbf{5 1 7}[\mathbf{6 \%}]$ \\
\hline
\end{tabular}

${ }^{a}$ Collected using a SH-SAW sensor with $0.8 \mu \mathrm{m}$ PIB coating.
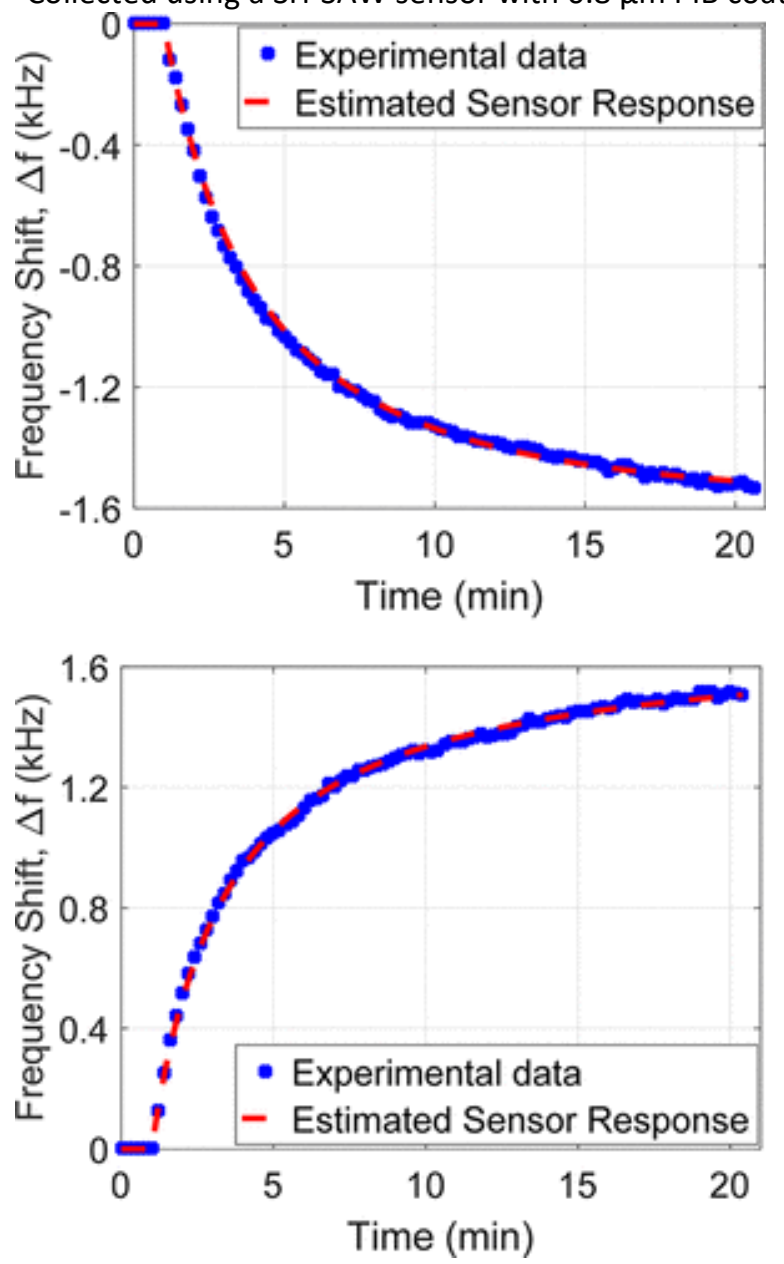

Figure 3. Measured response of a SH-SAW sensor with $0.8 \mu \mathrm{m}$ PIB coating to a solution of LNAPL in groundwater containing $400 \mathrm{ppb}$ benzene, $800 \mathrm{ppb}$ toluene, $550 \mathrm{ppb}$ ethylbenzene/xylenes, and unknown concentrations of interferents (top: sorption data, bottom: desorption data). Also shown (red dashed line) are the estimated sensor responses obtained using the five-analyte model. 
Multiple measurement data with BTEX concentrations in the low ppb to low ppm range were tested using the proposed technique. The results of this analysis are summarized in Figure 4 , which include results for sensor devices coated with $0.6 \mu \mathrm{m} \mathrm{PECH} \mathrm{(Figure} \mathrm{4a)} \mathrm{and} 0.8 \mu \mathrm{m}$ PIB (igure 4b). Also shown in Figure 4 are the relative percentage errors between the estimated concentrations and concentrations determined using GC-PID. Based on Figure 4, it can be observed that most of the estimated concentrations of BTEX compounds lie in close proximity to the ideal line (with an average of about $\pm 7 \%$ for benzene, $\pm 9 \%$ for toluene, and $\pm 11 \%$ for ethylbenzene and xylenes). Given the 7\% measurement error of the GC-PID instrument,(36) this implies the estimated concentrations are in excellent agreement with the concentrations determined using GC-PID. Note that among the BTEX compounds, the estimation errors for ethylbenzene and xylenes are the largest (in percent) due to the low concentration range (low ppb range) tested for these compounds. In the low ppb range, the signal noise limits the accuracy of the estimated concentrations. Another factor contributing to these inaccuracies might be the simplifying assumption made in modeling the combined response of interferents in the mixture using just two exponential terms. Also note that the estimation error for ethylbenzene and xylenes is not as critical as that for benzene, because the latter has a greater hazard potential and lower maximum contaminant level for drinking water. The estimated concentrations for benzene for both investigated sensor coatings are shown in Figure 5 . These results are found to be in excellent agreement with the concentrations determined using GC-PID.
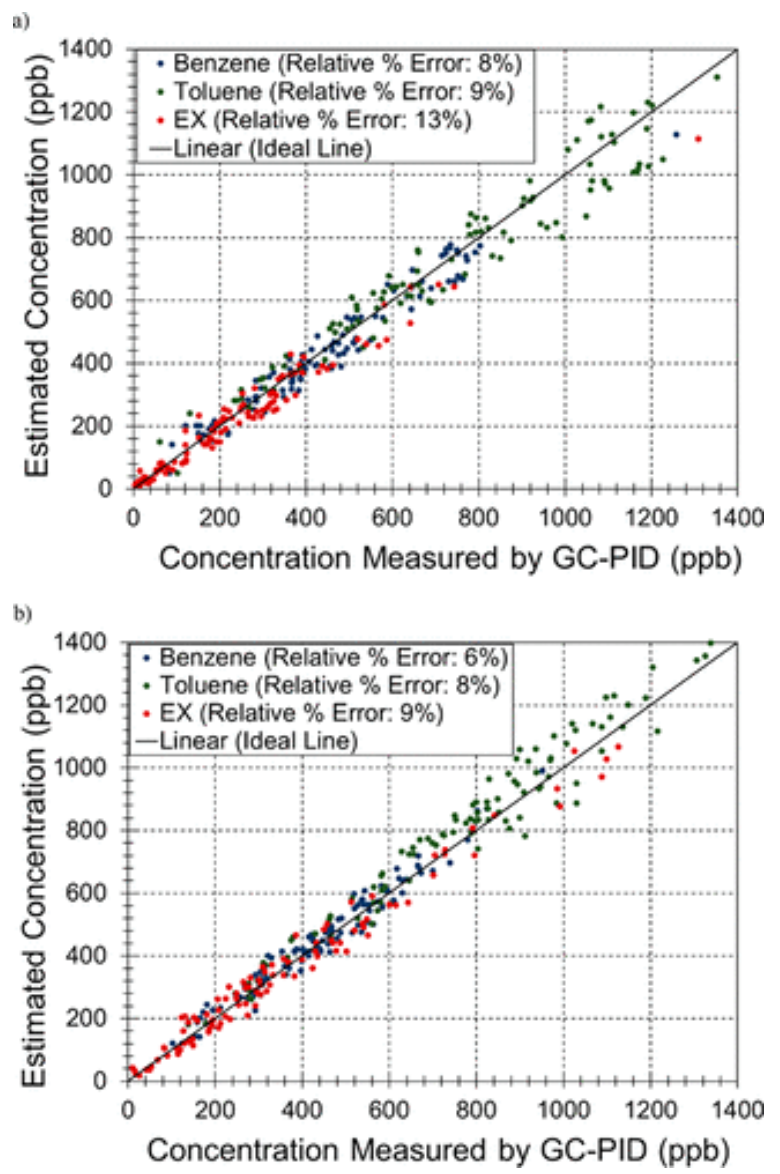

Figure 4. BTEX concentrations estimated using the proposed signal-processing technique and five-analyte model, obtained from SH-SAW sensors coated with $0.6 \mu \mathrm{m}$ PECH and $0.8 \mu \mathrm{m}$ PIB ( $a$ and $b$ ). Legends show the average percentage error between the estimated and the GC-PID measured concentrations. The diagonal line represents the ideal case (where estimated concentrations equal measured concentrations). 


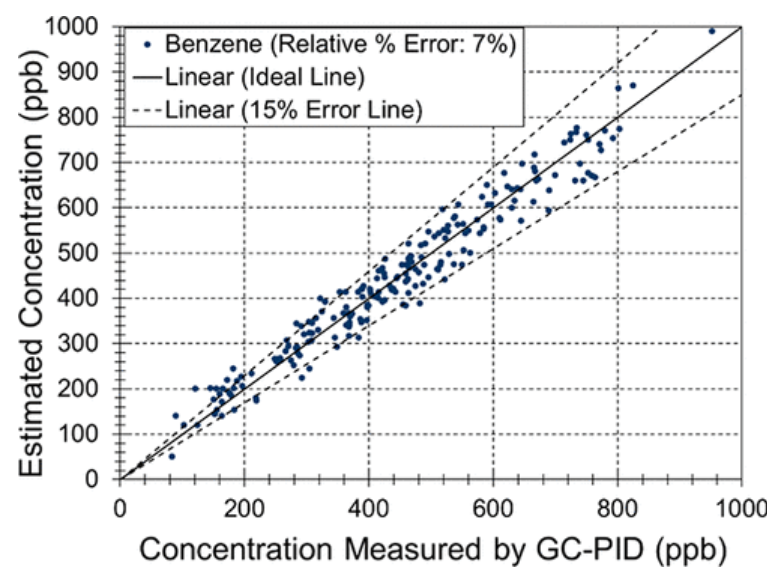

Figure 5. Benzene concentrations estimated using the proposed signal processing technique and five-analyte model, obtained from SH-SAW sensors coated with either $0.6 \mu \mathrm{m} \mathrm{PECH} \mathrm{or} 0.8 \mu \mathrm{m}$ PIB. Legends show the average percentage error of $\pm 7 \%$ between the estimated and the GC-PID measured concentrations. The diagonal line represents the ideal case (where estimated concentrations equal measured concentrations).

Utilizing the proposed approach, BTEX compounds in the sample can be identified and quantified in near-real time with high accuracy within approximately 2 min after data collection (including sorption and desorption responses). For applications which only require high accuracy for the estimated concentrations of benzene, even faster processing time can be achieved by just processing the sorption response data. In this case, benzene can be quantified with high accuracy in real time even before the sorption response reaches steadystate. $(23,25)$ Moreover, the accurate results obtained clearly highlight the potential of the proposed approach to detect BTEX compounds in aqueous phase using only the data collected from a single polymer-coated SHSAW device. If needed, in some applications, redundancy and improved detection limits could be achieved using the proposed approach with a small sensor array (consisting of 2 to 3 devices) coated with appropriately selected coatings.

\section{Summary and Conclusions}

The identification and quantification of target analytes (BTEX compounds) in the presence of interferents is demonstrated using an estimation-theory-based sensor signal-processing technique that utilizes data from only a single coated sensor device. The proposed approach is based on a multianalyte sensor response model and does not require any prior knowledge of the initial concentration range of the target analytes in the sample for accurately estimating the concentrations of those analytes of interest. The model utilizes a multivariate sensor parameter approach (in this case, equilibrium frequency shifts and response time constants from the singleanalyte responses to target analytes and the dominant interferents). Specifically, for the identification and quantification of BTEX analytes in the presence of interferents commonly found in groundwater, only a limited number of interferents must be considered to produce a response to the polymer-coated sensor: a five-analyte model is sufficient to analyze the six BTEX compounds/isomers along with the two most significant groups of interferents.

Two-step linear-estimation theory, comprising EW-RLSE and BKFs, is used to estimate the unknown parameters (i.e., equilibrium frequency shifts), which also exploits the time transients associated with sorption and desorption of the analytes. The proposed approach was tested extensively using measured responses of polymer-coated SH-SAW sensors to samples of LNAPL dissolved in water. Highly accurate estimates were obtained, with estimated concentrations for benzene, toluene, and ethylbenzene/xylenes falling within $\pm 7 \%$, 
$\pm 9 \%$, and $\pm 11 \%$, respectively, of the concentration determined using GC-PID. It is recalled here that the BTEX concentrations obtained from the GC-PID have an average error of $\pm 7 \%$.(36)

The approach presented here allows for more accurate detection and quantification of target analytes in nearreal time using the data from only a single coated sensor device, with high tolerance against measurement noise, and improves chemical speciation. This approach also can be implemented using a standard microcontroller to enable small, portable, cost-effective smart microsensor systems for various field applications including wastewater and groundwater monitoring, the monitoring of plumes in a subsurface marine oil spill, and spill clean-ups.

Finally, we point out that the proposed approach is independent of sensing platform, i.e., this signal-processing procedure can perform equally well with other sensing platforms such as chemiresistors, optical sensors, and MEMS-based sensors, provided the sensor responses using these platforms (1) offers the appropriate limits of detection and dynamic range and (2) can be analytically modeled. Our approach does not require a sensor array to achieve high accuracy in the individual quantification of multiple target analytes in a mixture, even in the presence of multiple interferents.

\section{Supporting Information}

The Supporting Information is available free of charge on the ACS Publications website at DOI: 10.1021 /acssensors.8b00353.

The authors declare no competing financial interest.

\section{Acknowledgments}

The authors would like to thank Nicholas Post for assistance in collecting some of the LNAPL measurement data, and Drs. Rachel E. Mohler and Ravi Kolhatkar for helpful discussion.

\section{References}

1 Albert, K. J.; Lewis, N. S.; Schauer, C. L.; Sotzing, G. A.; Stitzel, S. E.; Vaid, T. P.; Walt, D. R. Cross-reactive chemical sensor arrays. Chem. Rev. 2000, 100 (7), 2595, DOI: 10.1021/cr980102w

$\underline{2}$ Ricco, A. J.; Crooks, R. M.; Osbourn, G. C. Surface Acoustic Wave Chemical Sensor Arrays: New Chemically Sensitive Interfaces Combined with Novel Cluster Analysis to Detect Volatile Organic Compounds and Mixtures. Acc. Chem. Res. 1998, 31, 289-296, DOI: 10.1021/ar9600749

3 Bender, F.; Josse, F.; Ricco, A. J. Influence of Ambient Parameters on the Response of Polymer-Coated SHSurface Acoustic Wave Sensors to Aromatic Analytes in Liquid-Phase Detection. In Joint Conference of the IEEE IFCS and EFTF Proceedings, 2011; pp 422- 427.

$\underline{4}$ US Environmental Protection Agency: Underground Storage Tanks (USTs) [Online]; https://www.epa.gov/ust. $\underline{\mathbf{5}}$ What is BTEX and why is it important?, Aeroqual, 2017; http://www.aeroqual.com/what-is-btex. [Accessed: 22-Jun-2017].

$\underline{6}$ Bender, F.; Mohler, R.; Ricco, A. J.; Josse, F. Quantification of Benzene in Groundwater Using SH-Surface Acoustic Wave Sensors. IMCS Proc., 2012; pp 473476

7 MehIman, M. A.; Hemstreet, G. P.; Thorpe, J. J.; Weaver, N. K. Vol. VII: Renal effects of petroleum hydrocarbons; Princeton Scientific Publishers, Inc.: Princeton, NJ, 1984; pp 1- 23. 
8 US Environmental Protection Agency: National Primary Drinking Water

Regulations; https://www.epa.gov/ground-water-and-drinking-water/national-primary-drinking-waterregulations.

9 Lide, D. R. Aqueous Solubility and Henry's Law Constants of Organic Compounds. In CRC Handbook of Chemistry and Physics, 82nd ed.; CRC Press: Boca Raton, FL; pp 8-86- 8-112, 2001-2002.

10 Jones, Y. K.; Li, Z.; Johnson, M. M.; Josse, F.; Hossenlopp, J. M. ATR-FTIR Spectroscopic Analysis of Sorption of Aqueous Analytes Into Polymer Coatings Used With Guided SH-SAW Sensors. IEEE Sens. J. 2005, 5, 1175- 1184, DOI: 10.1109/JSEN.2005.859231

$\underline{11}$ US Environmental Protection Agency: Semiannual Report of UST Performance Measures, End Of Fiscal Year 2016; https://www.epa.gov/sites/production/files/2016-11/documents/ca-16-34.pdf.

12 Karlowatz, M.; Kraft, M.; Mizaikoff, B. Simultaneous Quantitative Determination of Benzene, Toluene, and Xylenes in Water Using Mid-Infrared Evanescent Field Spectroscopy. Anal. Chem. 2004, 76, 2643-2648, DOI: 10.1021/ac0347009

13 Schadle, T.; Pejcic, B.; Myers, M.; Mizaikoff, B. Fingerprinting Oils in Water via Their Dissolved VOC Pattern Using Mid-Infrared. Anal. Chem. 2014, 86, 9512-9517, DOI: 10.1021/ac5015029

14 Lima, K. M. G.; Raimundo, I. M., Jr.; Pimentel, M. F. Improving the detection limits of near infrared spectroscopy in the determination of aromatic hydrocarbons in water employing a silicone sensing phase. Sens. Actuators, B 2007, 125, 229- 233, DOI: 10.1016/j.snb.2007.02.008

15 Wittkamp, B. L.; Tilotta, D. C. Determination of BTEX Compounds in Water by Solid-Phase Microextraction and Raman Spectroscopy. Anal. Chem. 1995, 67, 600-605, DOI: 10.1021/ac00099a018

16 Kamal, M. A.; Klein, P. Estimation of BTEX in groundwater by using gas chromatography-mass spectrometry. Saudi J. Biol. Sci. 2010, 17, 205- 208, DOI: 10.1016/j.sjbs.2010.04.002

17 Silva, L. I. B.; Panteleitchouk, A. V.; Freitas, A. C.; Rocha-Santos, T. A. P.; Duarte, A. C. Microscale optical fibre sensor for BTEX monitoring in landfill leachate. Anal. Methods 2009, 1, 100-107, DOI: 10.1039/b9ay00077a

18 Ho, C. K.; Hughes, R. C. In-Situ Chemiresistor Sensor Package for Real-Time Detection of Volatile Organic Compounds in Soil and Groundwater. Sensors 2002, 2, 23- 24, DOI: 10.3390/s20100023

19 Cooper, J. S.; Raguse, B.; Chow, E.; Hubble, L.; Muller, K.-H.; Wieczorek, L. Gold Nanoparticle Chemiresistor Sensor Array that Differentiates between Hydrocarbon Fuels Dissolved in Artificial Seawater. Anal. Chem. 2010, 82, 3788-3795, DOI: 10.1021/ac1001788

$\underline{20}$ Cooper, J. S.; Kiiveri, H.; Hubble, L. J.; Chow, E.; Webster, M. S.; Muller, K.-H.; Sosa-Pintos, A.; Bendavid, A.; Raguse, B.; Wieczorek, L. Quantifying BTEX in aqueous solutions with potentially interfering hydrocarbons using a partially selective sensor array. Analyst 2015, 140, 3233- 3238, DOI: 10.1039/C5AN00223K

$\underline{21}$ Bender, F.; Mohler, R. E.; Ricco, A. J.; Josse, F. Identification and Quantification of Aqueous Aromatic Hydrocarbons Using SH-Surface Acoustic Wave Sensors. Anal. Chem. 2014, 86, 1794- 1799, DOI: 10.1021/ac403724f

$\underline{22}$ Bender, F.; Josse, F.; Mohler, R. E.; Ricco, A. J. Design of SH-Surface Acoustic Wave Sensors for Detection of ppb Concentrations of BTEX in Water. Joint UFFC. EFTF and PFM Symposium 2013, 628-631, DOI: 10.1109/EFTF-IFC.2013.6702067

$\underline{23}$ Sothivelr, K.; Bender, F.; Josse, F.; Ricco, A. J.; Yaz, E. E.; Mohler, R. E.; Kolhatkar, R. Detection and Quantification of Aromatic Hydrocarbon Compounds in Water Using SH-SAW Sensors and EstimationTheory-Based Signal Processing. ACS Sens. 2016, 1, 63- 72, DOI: 10.1021/acssensors.5b00024

$\underline{24}$ Reif, K.; Gunther, S.; Yaz, E.; Unbehauen, R. Stochastic Stability of the Discrete-Time Extended Kalman Filter. IEEE Trans. Autom. Control 1999, 44, 714- 728, DOI: 10.1109/9.754809

$\underline{25}$ Sothivelr, K.; Bender, F.; Josse, F.; Yaz, E. E.; Ricco, A. J.; Mohler, R. E. Online Chemical Sensor Signal Processing Using Estimation Theory: Quantification of Binary Mixtures of Organic Compounds in the 
Presence of Linear Baseline Drift and Outliers. IEEE Sens. J. 2016, 16, 750- 761, DOI: 10.1109/JSEN.2015.2485784

26 Bender, F.; Kim, C.; Mlsna, T.; Vetelino, J. F. Characterization of a $\mathrm{WO}_{3}$ thin film chlorine sensor. Sens. Actuators, B 2001, 77, 281- 286, DOI: 10.1016/S0925-4005(01)00711-0

27 Taylor, G. Dispersion of Soluable Matter in Solvent Flowing Slowly through a Tube. Proc. R. Soc. London, Ser. A 1953, 219, 186-203, DOI: 10.1098/rspa.1953.0139

28 Vaseghi, S. V. Multimedia Signal Processing: Theory and Applications in Speech, Music and Communications; John Wiley \& Sons, Ltd: West Sussex, London, 2007.

$\underline{29}$ Goodwin, G. C.; Sin, K. S. Adaptive Filtering Prediction and Control; Dover Publications, Inc: Mineola, NY, 2009.

30 Lewis, F. L.; Xie, L.; Popa, D. Optimal and Robust Estimation: With an Introduction to Stochastic Control Theory, 2nd ed.; CRC Press: Boca Raton, FL, 2008.

31 Anderson, B. D. O.; Moore, J. B. Optimal Filtering; Prentice-Hall: Englewood Cliffs, NJ, 1979.

32 Kalman, R. E. A New Approach to Linear Filtering and Prediction Problems. J. Basic Eng. 1960, 82, 35- 45, DOI: 10.1115/1.3662552

33 Simon, D. Optimal State Estimation: Kalman, $H_{\infty}$, and Nonlinear Approaches; Wiley-Interscience: Hoboken, NJ, 2006.

34 Bender, F.; Mohler, R. E.; Ricco, A. J.; Josse, F. Analysis of Binary Mixtures of Aqueous Aromatic Hydrocarbons with Low-Phase-Noise Shear-Horizontal Surface Acoustic Wave Sensors Using Multielectrode Transducer Designs. Anal. Chem. 2014, 86, 11464-11471, DOI: 10.1021/ac503701b

35 Josse, F.; Bender, F.; Cernosek, R. W. Guided Shear-Horizontal Surface Acoustic Wave Sensors for Chemical and Biochemical Detection in Liquids. Anal. Chem. 2001, 73, 5937-5944, DOI: 10.1021/ac010859e

36 A Micro-GC Based Chemical Analysis System; Defiant Technologies, Inc., 2014; http://www.defianttech.com/pdfs/Pittcon\%202014\%20A\%20Micro-GC\%20Based\%20Chemical\%20Analysis\%20System.pdf. 